\title{
One Loop Partition Function in Plane Waves R-R Background
}

\author{
Amine B. Hammou \\ Department of Physics and Institute of Plasma Physics, \\ University of Crete, and FO.R.T.H. 71003 Heraklion GREECE
}

\begin{abstract}
We compute the one loop partition function of type IIB string in plane wave R-R 5-form background $F^{5}$ using both path integral and operator formalisms and show that the two results agree perfectly. The result turns out to be equal to the partition function in the flat background. We also study the Tadpole cancellation for the unoriented closed and open string model in plane wave R-R 5-form background studied in hep-th/0203249 and find that the cancellation of the Tadpole requires the gauge group to be $\mathrm{SO}(8)$.
\end{abstract}

Keywords: Superstrings, Partition function, pp-waves

email address: amine@physics.uoc.gr 


\section{Introduction}

Recently a new maximally supersymmetric solution of type IIB supergravity was found [1] and it was related by a special limit (Penrose limit) to the famous $A d S_{5} \times S^{5}$ [2]. It turns out to be a ten dimensional plane wave space with metric and R-R 5-form flux

$$
\begin{aligned}
d s^{2} & =-2 d x^{+} d x^{-}-\mu^{2} x_{i} x^{i}\left(d x^{+}\right)^{2}+d x_{i} d x^{i}, \\
F_{+1234} & =F_{+5678}=2 \mu .
\end{aligned}
$$

Remarkably, the Green-Schwarz formulation of the theory shows up to be exactly solvable in the light-cone gauge [3, 4]. Moreover, the exact string spectrum in plane wave has been successfully compared to the dual $\mathcal{N}=4$ gauge theory operators [5]. A similar comparison has been carried out in [6] for a plane wave model dual to an $\mathcal{N}=2 S p(N)$ gauge theory with a hypermultiplet in the antisymmetric representation and four fundamental hypermultiplets. This theory is dual to string theory on $A d S_{5} \times S^{5} / Z_{2}$, where the $Z_{2}$ is an orientifold action [7]. In this paper we will consider this model and study its anomaly cancellation -Tadpoles-, this is important for the consistency of the theory, and to fix the gauge group which was crucial in the comparison with the dual $\mathcal{N}=2$ gauge theory [6]. The paper is organized as follows. Section 2 we briefly review the light cone gauge world sheet theory of type IIB strings in plane wave R-R background. In section 3 we compute the partition function using both path integral and operator formalisms and show that the two results agree and are equal to the partition function in flat space background. Finally in section 4 we will study the anomaly cancellation -Tadpoles- for a model of unoriented closed and open strings in plane wave R-R background considered in [6].

\section{Review of type IIB in plane wave R-R background}

The $\kappa$-symmetry gauge fixed type IIB GS Lagrangian in the plane wave $\mathrm{R}$ - $\mathrm{R}$ background i.e. $\bar{\gamma}^{+} \theta=\bar{\gamma}^{+} \bar{\theta}=0$ is given by 33 :

$$
\begin{aligned}
\mathcal{L}_{B} & =-\frac{1}{2} \sqrt{g} g^{a b}\left(2 \partial_{a} x^{+} \partial_{b} x^{-}-x_{i}^{2} \partial_{a} x^{+} \partial_{b} x^{+}+\partial_{a} x^{i} \partial_{b} x^{i}\right) \\
\mathcal{L}_{F} & =-\mathrm{i} \sqrt{g} g^{a b} \partial_{b} x^{+}\left(\bar{\theta} \bar{\gamma}^{-} \partial_{a} \theta+\theta \bar{\gamma}^{-} \partial_{a} \bar{\theta}+2 \mathrm{i} \partial_{a} x^{+} \bar{\theta} \bar{\gamma}^{-} \Pi \theta\right) \\
& +\epsilon^{a b} \partial_{a} x^{+}\left(\theta \bar{\gamma}^{-} \partial_{b} \theta+\bar{\theta} \bar{\gamma}^{-} \partial_{b} \bar{\theta}\right) .
\end{aligned}
$$

where $i=1, \ldots, 8$ and $\Pi=\gamma^{1} \bar{\gamma}^{2} \gamma^{3} \bar{\gamma}^{4}$. As in flat space case we can eliminate the $\partial x^{+}$-factors from the kinetic terms of the fermionic fields. This is possible by choosing the bosonic light-cone gauge which fixes the residual conformal symmetry

$$
\sqrt{g} g^{a b}=\eta^{a b}, \quad x^{+}(\tau, \sigma)=\alpha^{\prime} p^{+} \tau .
$$


The resulting Lagrangian will be that of 8 free massive two dimensional bosons and 8 free massive Majorana two dimensional fermionic fields propagating in a flat two dimensional space with mass $m=\alpha^{\prime} \mu p^{+}$. Note that we can recover the flat case in the limit $\mu \rightarrow 0$, while the limit $\alpha^{\prime} p^{+} \rightarrow 0$ corresponds to supergravity in the plane wave background [3].

The Light-cone superstring Hamiltonian can be expressed ast:

$$
\begin{aligned}
H= & \mu\left(a_{0}^{i \dagger} a_{0}^{i}+S_{0}^{a \dagger} S_{0}^{a}\right) \\
& +\frac{1}{p^{+}} \sum_{n=1}^{\infty} \omega_{n}\left(a_{n}^{i \dagger} a_{n}^{i}+\tilde{a}_{n}^{i \dagger} \tilde{a}_{n}^{i}+S_{n}^{a \dagger} S_{n}^{a}+\tilde{S}_{n}^{a \dagger} \tilde{S}_{n}^{a}\right),
\end{aligned}
$$

where

$$
\omega_{n}=\sqrt{m^{2}+n^{2}}
$$

and we are summing over the repeated indices $i$ and $a$ going from $1, \ldots, 8$. The bosonic zero modes are defined through

$$
a_{0}^{i}=\frac{1}{\sqrt{2 m}}\left(p_{0}^{i}+i m x_{0}^{i}\right), \quad a_{0}^{i+}=\frac{1}{\sqrt{2 m}}\left(p_{0}^{i}-i m x_{0}^{i}\right),
$$

$p_{0}^{i}$ and $x_{0}^{i}$ are the zero modes of the bosonic fields, which in the flat limit will correspond to the momentum and the center of mass positions. The fermionic fields $S$ and $\tilde{S}$ are related to the fields $\theta$ and $\bar{\theta}$ in the same way as in the flat space. The vacuum state is defined as the direct product of the zero mode vacuum and the vacuum of the string oscillation modes annihilated by all the annihilation operators appearing in the Hamiltonian (2.4). Generic Fock space states are then obtained by acting on the vacuum with the creation operators and the subspace of physical states is obtained by imposing the level matching condition i.e. $N=\tilde{N}$ with

$$
N=\sum_{n=1}^{\infty} n\left(a_{n}^{i \dagger} a_{n}^{i}+S_{n}^{a \dagger} S_{n}^{a}\right)
$$

and the same expression for $\tilde{N}$ with tilde on the oscillators.

\section{Closed String Partition function}

In this section we will compute the partition function of type IIB superstring in plane wave $\mathrm{R}-\mathrm{R}$ background described above, using the path integral formalism $\mathrm{l}$ and show that the result is zero as it should be for any globally supersymmetric background. Surprisingly, it turns out that the partition function of type IIB in plane wave R-R background is equal to the partition function of type IIB in flat background. This result seems to contradict the result obtained in [9] using the operator formalism. We will show that in the operator formalism the partition

\footnotetext{
${ }^{1}$ We will put $\alpha^{\prime}=1$ in what follows, then all one needs to do to restore the dependence on the string tension is to rescale $p^{+} \rightarrow \alpha^{\prime} p^{+}[3]$.

${ }^{2}$ I would like to thank very much A.A. Tseytlin for correspondence on this point [8].
} 
function computed by Takayanagi [9] is also zero after a careful integration over $p^{+}$and $p^{-}$. This is to be expected since the two results can be compared only after performing the integral over the momentum.

\subsection{Path Integral}

Following Kallosh and Morozov [10] [ the partition function in the path integral formalism can be computed by fixing the semi-light cone gauge i.e. $\bar{\gamma}^{+} \theta=\bar{\gamma}^{+} \bar{\theta}=0$ and $g_{a b}=\rho g_{a b}^{(m)}$, where

$g_{a b}^{(m)}$ is some background metric. In conformal coordinates $z, \bar{z}$ with $g_{z z}^{(m)}=g_{\bar{z} \bar{z}}^{(m)}=0, g_{z \bar{z}}^{(m)}=\frac{1}{2}$ omitting the integration over the background moduli, the gauge fixed path integral will take the form

$$
\int D x^{\mu} D \bar{\theta} D \theta D b D c\left(\operatorname{Det}_{\bar{z}}\right)^{-8} e^{-S-S_{g h}},
$$

where $u_{\bar{z}}=\partial_{\bar{z}} x^{+}$and the action $S$ stand for the covariant action associated to the bosonic and fermionic Lagrangian (2.1)-(2.2) expressed in conformal coordinates $z, \bar{z}$

$$
\begin{aligned}
S & =\int d^{2} z\left[2 \partial_{z} x^{\mu} \partial_{\bar{z}} x_{\mu}-x_{i}^{2} \partial_{z} x^{+} \partial_{\bar{z}} x^{+}\right. \\
& \left.+\mathrm{i} \partial_{\bar{z}} x^{+}\left(\theta^{1} \bar{\gamma}^{-} \partial_{z} \theta^{1}+\theta^{2} \bar{\gamma}^{-} \partial_{z} \theta^{2}+4 \mathrm{i} \partial_{z} x^{+} \theta^{1} \bar{\gamma}^{-} \Pi \theta^{2}\right)\right],
\end{aligned}
$$

where $\mu=+,-, i$ and $\theta^{1}=\frac{1}{\sqrt{2}}(\theta+\bar{\theta}), \theta^{2}=-\frac{i}{\sqrt{2}}(\theta-\bar{\theta})$ are two real Majorana-Weyl spinors which would correspond to the left and right moving fermions in the flat background limit. The ghosts action is as usual given by

$$
S_{g h}=\int d^{2} z\left(b \partial_{\bar{z}} c+\bar{b} \partial_{z} \bar{c}\right) .
$$

The $\left(\operatorname{Detu}_{\bar{z}}\right)^{8}$ is the local measure of the integration related to the Faddeev-Popov fermionic gauge symmetry nonpropagating ghosts. It provides the independence of the theory of the way in which local fermionic gauge symmetry, $\kappa$-symmetry, was fixed. Using the fact that $S O(8)$ spinors $\left(\bar{\gamma}^{+} \theta^{\mathcal{I}}=0\right)$ may be presented as two $S U(4)$ spinors $\left(\eta_{\mathcal{I}}^{k}, \theta_{k}^{\mathcal{I}}\right), k=1, \ldots, 4$ and $\mathcal{I}=1,2$. Then $\theta^{\mathcal{I}} \gamma^{-} u_{\bar{z}} \partial_{z} \theta^{\mathcal{I}} \rightarrow \eta_{\mathcal{I}}^{k} u_{\bar{z}} \partial_{z} \theta_{k}^{\mathcal{I}}+\frac{1}{2} \eta_{\mathcal{I}}^{k} \theta_{k}^{\mathcal{I}} \partial_{z} u_{\bar{z}}$. The last term $\eta_{\mathcal{I}}^{k} \theta_{k}^{\mathcal{I}} \partial_{z} \partial_{\bar{z}} x^{+}$can be absorbed into a redefinition of $x^{-}$. We can absorb the factor $u_{\bar{z}}$ in a redefinition of the spinors $\eta_{\bar{z}, \mathcal{I}}^{k}=$ $\eta_{\mathcal{I}}^{k} \partial_{\bar{z}} x^{+}$, then the Jacobian of this transformation will cancel the factor $\left(\operatorname{Det}_{\bar{z}}\right)^{8}$ appearing in the measure (3.1). We can now perform the integration over $x^{-}$which will give a delta function' $\delta\left(\partial_{z} \partial_{\bar{z}} x^{+}\right)$, and over $x^{+}$which will first constrain $x^{+}$to be constant on the Torus i.e.

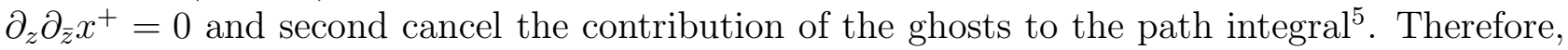
all the terms containing $\partial_{z} x^{+}$in the action will cancel. These are the terms which was the

\footnotetext{
${ }^{3}$ I am very grateful to A.A. Tseytlin for drawing my attention to this paper.

${ }^{4}$ We are considering a Euclidean space time by means of a wick rotation, hence we should integrate our final result over a Euclidean torus [11].

${ }^{5}$ as usual the contribution of the ghost to the path integral will cancel the same contribution coming from the bosonic fields in the light-cone directions.
} 
source for the mass in the bosonic light-cone gauge theory discussed in the previous section. The path integral will become

$$
\int D x^{i} D \eta_{\bar{z}, \mathcal{I}}^{k} D \theta_{k}^{\mathcal{I}} \exp \left\{-\int d^{2} z\left(\partial_{z} x^{i} \partial_{\bar{z}} x^{i}+\eta_{\bar{z}, \mathcal{I}}^{k} \partial_{z} \theta_{k}^{\mathcal{I}}\right)\right\}
$$

which is nothing but the path integral of the bosonic and fermionic fields in the transverse directions. Therefore, the partition function is zero (as it should be due to supersymmetry) due to fermionic zero modes [8] and it is equal to the partition function of type IIB string theory in flat background.

\subsection{Operator Formalism}

Now we would like to do the same computation using the Operator formalism, and show that it leads to the same result. Following Takayanagi [9] we define the partition function as

$$
Z=\int_{\mathcal{F}} \frac{d \tau_{1} d \tau_{2}}{\tau_{2}} \int d p^{+} d p^{-} \operatorname{Tr}(-1)^{F} e^{-2 \pi \tau_{2} p^{+}\left(p^{-}+H\right)+2 \pi i \tau_{1}(N-\tilde{N})},
$$

where $H$ is the light-cone Hamiltonian (2.4), $N$ and $\tilde{N}$ are the number operators (2.7). Tr denotes the trace in the Hilbert space of the light-cone string theory and $F$ is the fermion number operator. The modular invariance of the partition function enables us to restrict the integration of the torus moduli $\tau, \bar{\tau}$ to the fundamental region denoted $\mathcal{F}$.

Performing the trace over the Hilbert space we find

$$
Z_{\mathrm{IIB}}=\int_{\mathcal{F}} \frac{d \tau_{1} d \tau_{2}}{\tau_{2}} \int d p^{+} d p^{-} e^{-2 \pi \tau_{2} p^{+} p^{-}}\left(\frac{Z_{f}\left(\tau_{1}, \tau_{2} \mid m\right)}{Z_{B}\left(\tau_{1}, \tau_{2} \mid m\right)}\right)^{4},
$$

where $Z_{f}$ and $Z_{B}$ are the partition functions of a massive fermion and boson respectively with mass $m=\mu p^{+} \neq 0$ given by

$$
\begin{gathered}
Z_{f, B}(\tau, \bar{\tau} \mid m)=e^{4 \pi \tau_{2} \Delta_{(m)}}\left(1-e^{-2 \pi \tau_{2} m}\right)^{2} \prod_{n=1}^{\infty}\left(1-e^{-2 \pi \tau_{2} \sqrt{m^{2}+n^{2}}+2 \pi i \tau_{1} n}\right)^{2} \\
\times\left(1-e^{-2 \pi \tau_{2} \sqrt{m^{2}+n^{2}}-2 \pi i \tau_{1} n}\right)^{2} .
\end{gathered}
$$

The factor $\Delta_{(m)}$ corresponds to the zero point energy defined by

$$
\Delta_{(m)}=-\frac{1}{2 \pi^{2}} \sum_{p=1}^{\infty} \int_{0}^{\infty} d s e^{-p^{2} s-\frac{\pi^{2} m^{2}}{s}}
$$

and in the massless limit it reproduces the known value $-1 / 12$. It is easy to show that (3.6) is modular invariant provided we also change the momentum $p^{+}$and $p^{-}$to $|\tau| p^{+}$and $|\tau| p^{-}$ respectively [9]. Finally to compare the result of the partition function in the two formalisms we should perform the integration over $p^{+}$and $p^{-}$and since we are doing the computation in the Euclidean space they are complex variables. Since the integrand is a function of only $p^{+}$ then the integral will be the integrand valued at $p^{+}=0$. The functions $Z_{f}$ and $Z_{B}(3.7)$ are 
equal if $p^{+} \neq 0$ but in the $p^{+} \rightarrow 0$ limit they are different, and the difference comes from the zero mode part. In fact, the limit $p^{+} \rightarrow 0$ of $p^{+} H$ will reproduce exactly the partition function of type IIB in the flat background. The factor $\left(1-e^{-2 \pi \tau_{2} m}\right)^{8}$ in $Z_{f}$ will goes to zero reproducing the $(8-8)^{2}$ of the flat background limit. Whereas, in $Z_{B}$ using (2.6) it is easy to see that the trace over the bosonic zero mode part in the limit $p^{+} \rightarrow 0$ will just become an integration over non-compact momentum $p_{0}^{i}$ which now become continue, reproducing the familiar factor of $1 / \tau_{2}^{4}$. Therefore, we will find

$$
Z_{\mathrm{IIB}}=\int_{\mathcal{F}} \frac{d \tau_{1} d \tau_{2}}{\tau_{2}^{6}} \frac{\theta_{1}^{4}(\tau)}{\eta^{12}(\tau)} \frac{\bar{\theta}_{1}^{4}(\bar{\tau})}{\bar{\eta}^{12}(\bar{\tau})} .
$$

It is worth noting that the limit $p^{+} \rightarrow 0$ of $H$ is not without ambiguities but what we have really done is the limit $p^{+} \rightarrow 0$ of $p^{+} H$ which is well defined and it corresponds to the flat background limit in the G-S formulation. Similar observations was made in [12 where the torus partition function of the WZW model for a non semi-simple group was shown to be equal to the flat space value. Following the same arguments as for the R-R 5-form background we can show that the partition function in the R-R 3-form backgrounds of [4] is also trivial

\section{Open string in Plane wave R-R background}

In this section we will start by giving a brief review of the model of unoriented closed and open strings in plane wave $\mathrm{R}$ - $\mathrm{R}$ background considered in [6] and study its Tadpole cancellation. We will show that the model is free of anomalies if the gauge group is $S O(8)$

\subsection{Review of open string model}

The model studied in [6] consist of the $O(7)$ orientifold projection of the plane wave solution of 10d type IIB supergravity. Since the $O(7)$ plane carries -4 units of D7-brane charge, we should add 4 D7 branes to cancel the charge locally. This will produce an $S O(8)$ gauge group on the world volume of the D7 branes. An other way to obtain this system is to start with type IIB theory compactified on $S^{1} \times S^{1}$ with $x^{7,8}$-coordinates, then project by the world-sheet parity $\Omega$ which gives type I theory. Do two T-dualities on the compact directions $x^{7,8}$, this will change D9 branes to D7 branes transverse to the compact directions. The resulting theory is type IIB projected out by $\Omega(-1)^{F_{L}} R$, where $F_{L}$ is the left fermion number and $R$ is a $Z_{2}$ projection $x^{7,8} \rightarrow-x^{7,8}$. There are four fixed points where the orientifold planes are siting, so we should add the D7 branes to cancel locally and globally the total charge. Unless type I theory, this model have D3 branes in its BPS spectrum. As in type IIB theory we can study the Penrose limit of the near horizon geometry of a D3 brane in this model (O7-D7-D3)[6]. Since,

\footnotetext{
${ }^{6}$ For the case of NS-NS 3-form background it was argued in [4] that the partition function vanishes by virtue of supersymmetry of the plane wave background and is the same as the flat space partition function.

${ }^{7}$ When this work was nearing completion there appeared a paper 15 where the authors analysed the Tadpole condition in an orientifold of type IIB string theory in the plane wave background supported by null R-R 3-form flux $F^{3}$, so we will some times refer to it.
} 
the orientifold is parallel to the light cone directions, the theory in this background result in an exactly solvable string theory.

\section{Closed Strings}

The closed sector of this model is the same as type IIB theory in plane wave R-R background. The light cone Hamiltonian is given by (2.4). The $Z_{2}$ projection $\Omega(-1)^{F_{L}} R$ acts on the bosonic and fermionic oscillator modes as

$$
\begin{aligned}
& a_{n}^{i} \rightarrow \tilde{a}_{n}^{i} i=1, \ldots, 6 \quad ; a_{n}^{i} \rightarrow-\tilde{a}_{n}^{i} i=7,8 \\
& S_{n} \rightarrow i \gamma^{56} \tilde{S}_{n}
\end{aligned}
$$

where the action of $\gamma^{56}$ will be to give a minus sign to half of the fermionic oscillator modes.

\section{Open Strings}

There are also open strings that are stretched between the $D 7$ branes. In the Penrose limit under consideration these branes are located at the origin of the 78-plane meaning that $x^{7}$ and $x^{8}$ should satisfy Dirichlet boundary conditions at $\sigma=0, \pi$

$$
\begin{aligned}
& \partial_{\sigma} x^{i}=0 \quad i=1, \ldots, 6 ; \quad x^{i}=0 \quad i=7,8 \\
& S=\gamma^{78} \tilde{S}
\end{aligned}
$$

The light cone open string Hamiltonian is given by

$$
H=\frac{1}{2}\left(\mu\left(a_{0}^{I \dagger} a_{0}^{I}+S_{0}^{A \dagger} S_{0}^{A}+2\right)+\frac{1}{p^{+}} \sum_{n=1}^{\infty} \omega_{n}\left(a_{n}^{i \dagger} a_{n}^{i}+S_{n}^{a \dagger} S_{n}^{a}\right)\right),
$$

where as before $i, a=1, \ldots, 8$, while $I=1, \ldots, 6$ and $A=1, \ldots, 4$. Note that the difference with respect to the closed string case is that here we have 6 Neumann directions $x^{I}$ which have zero modes, and the two Dirichlet directions instead will not have zero modes. For the fermions there are in the other hand 8 zero modes of which 4 of them are creation and 4 annihilation operators, which is reflecting the fact that now we have half of the original type IIB supersymmetry. Finally the factor of 2 in the zero mode part of the Hamiltonian (4.2) is the vacuum energy due to the mismatch between the bosonic and fermionic zero modes. Finally, the orientifold $Z_{2}$ projection on the different bosonic and fermionic oscillator modes is

$$
a_{n}^{i} \rightarrow(-1)^{n} a_{n}^{i}, \quad i=1, \cdots, 8 ; \quad S_{n} \rightarrow(-1)^{n} S_{n}
$$

\subsection{Tadpole Cancellation}

Let us proceed now and study the anomaly cancellation in this unoriented closed and open string model by computing the relevant vacuum amplitudes with zero Euler Character. These are the Klein Bottle, the Annulus and the Mobius Strip?.

\footnotetext{
${ }^{8}$ For a recent review of Open Strings and Tadpole cancellation see [14] and references therein.
} 
The amplitude can be expressed in terms of the modified modular $f$ functions [13]:

$$
\begin{aligned}
& f_{1}^{(m)}(q)=q^{-\frac{1}{2} \Delta_{(m)}}\left(1-q^{m}\right)^{\frac{1}{2}} \prod_{n=1}^{\infty}\left(1-q^{\sqrt{m^{2}+n^{2}}}\right) \\
& f_{2}^{(m)}(q)=q^{-\frac{1}{2} \Delta_{(m)}}\left(1+q^{m}\right)^{\frac{1}{2}} \prod_{n=1}^{\infty}\left(1+q^{\sqrt{m^{2}+n^{2}}}\right) \\
& f_{3}^{(m)}(q)=q^{-\frac{1}{2} \Delta_{(m)}^{\prime}} \prod_{n=1}^{\infty}\left(1+q^{\sqrt{m^{2}+\left(n-\frac{1}{2}\right)^{2}}}\right) \\
& f_{4}^{(m)}(q)=q^{-\frac{1}{2} \Delta_{(m)}^{\prime}} \prod_{n=1}^{\infty}\left(1-q^{\sqrt{m^{2}+\left(n-\frac{1}{2}\right)^{2}}}\right)
\end{aligned}
$$

where $q=e^{-2 \pi t}, \Delta_{(m)}$ was given in (3.8) and $\Delta_{(m)}^{\prime}$ is given by

$$
\Delta_{(m)}^{\prime}=-\frac{1}{2 \pi^{2}} \sum_{p=1}^{\infty}(-1)^{p} \int_{0}^{\infty} d s e^{-p^{2} s-\frac{\pi^{2} m^{2}}{s}} .
$$

Note that in the limit $m \rightarrow 0$ these function can be expressed in terms of standard $\theta$ functions and $\Delta_{(m)} \rightarrow-1 / 12$ and $\Delta_{(m)}^{\prime} \rightarrow 1 / 24$.

Under modular transformation $l=1 / t$ they transform as follows:

$$
f_{1}^{(m)}(q)=f_{1}^{(\tilde{m})}(\tilde{q}), \quad f_{2}^{(m)}(q)=f_{4}^{(\tilde{m})}(\tilde{q}), \quad f_{3}^{(m)}(q)=f_{3}^{(\tilde{m})}(\tilde{q})
$$

where $\tilde{m}=m / l$ and $\tilde{q}=e^{-2 \pi l}$.

\section{Klein Bottle}

The Klein Bottle amplitude in the direct channel is given by the Torus amplitude (3.5) with the $Z_{2}$ projections (4.1) and leads

$$
Z_{\mathcal{K}}=\frac{1}{2} \int \frac{d \tau_{2}}{\tau_{2}} \int d p^{+} d p^{-} e^{-2 \pi \tau_{2} p^{+} p^{-}} \mathcal{K}\left(\tau_{2} \mid m\right),
$$

where $\mathcal{K}$ is given by

$$
\mathcal{K}\left(\tau_{2} \mid m\right)=\frac{\left(1+q^{\frac{m}{2}}\right)^{2}}{\left(1-q^{\frac{m}{2}}\right)^{2}}\left(\frac{f_{1}^{(m)}(q)}{f_{1}^{(m)}(q)}\right)^{8}
$$

with $t=2 \tau_{2}$. We can easily see that the Klein Bottle amplitude vanish by supersymmetry after an integration over $p^{+}$and $p^{-}$as it happens for the Torus amplitude. In the limit $p^{+} \rightarrow 0$ we find from the fermionic zero modes $(8-8)$ i.e. 16 supersymmetries. Whereas, from the bosonic zero modes we get a factor of $1 / \tau_{2}^{3}$ by integration over the momentum in the six Neumann directions. We don't see the Dirichlet directions due to the fact that we are siting 
on the point $x^{7,8}=0$. To extract the ultraviolet behavior of the Klein Bottle amplitude we should go to the transverse channel. This is done by modular transformation $l=1 / t$. As in the closed string case the modular transformation will change at the same time $p^{+}$and $p^{-}$now to $p^{+}=p^{+} / l$ and $p^{--}=p^{-}$. The modular transformed amplitude is '

$$
\tilde{\mathcal{K}}(l \mid \tilde{m})=\frac{\left(1+e^{-\pi \tilde{m}}\right)^{2}}{\left(1-e^{-\pi \tilde{m}}\right)^{2}}\left(\frac{f_{1}^{(\tilde{m})}(\tilde{q})}{f_{1}^{(\tilde{m})}(\tilde{q})}\right)^{8}
$$

where $\tilde{m}=\mu p^{+}$. Putting every thing together we find $\int_{0}^{\infty} d l$ times

$$
\tilde{Z}_{\mathcal{K}}=\frac{1}{2} \int d p^{\prime+} d p^{\prime-} e^{-\pi p^{\prime+} p^{\prime-}} \frac{\left(1+e^{-\pi \mu p^{\prime+}}\right)^{2}}{\left(1-e^{-\pi \mu p^{\prime+}}\right)^{2}}\left(\frac{f_{1}^{(\tilde{m})}(\tilde{q})}{f_{1}^{(\tilde{m})}(\tilde{q})}\right)^{8}
$$

\section{Annulus}

In this case the trace is over open string spectrum and in order to account for the internal degrees of freedom (Chan-Paton factors) we associate a multiplicity $N$ to each of the string ends. The direct channel amplitude can be computed easily using the open string Hamiltonian (4.2) leading

$$
Z_{\mathcal{A}}=\frac{N^{2}}{2} \int \frac{d \tau_{2}}{\tau_{2}} \int d p^{+} d p^{-} e^{-2 \pi \tau_{2} p^{+} p^{-}} \mathcal{A}\left(\tau_{2} \mid m\right)
$$

where $\mathcal{A}$ is given by

$$
\mathcal{A}\left(\tau_{2} \mid m\right)=\frac{q^{-m}}{\left(1-q^{m}\right)^{2}}\left(\frac{f_{1}^{(m)}(q)}{f_{1}^{(m)}(q)}\right)^{8}
$$

with $t=\tau_{2} / 2$ for the Annulus. As for the Klein Bottle the Annulus amplitude also vinishes by integration over the $p^{ \pm}$momentum. Making a modular transformation to go to the transverse channel $l=1 / t$ the momentum $p^{+}$and $p^{-}$get changed to $p^{+}=p^{+} / l$ and $p^{\prime-}=4 p^{-}$. In terms of $\tilde{q}$ the amplitude will lead

$$
\tilde{\mathcal{A}}(l \mid \tilde{m})=\frac{e^{-2 \pi \tilde{m}}}{\left(1-e^{-2 \pi \tilde{m}}\right)^{2}}\left(\frac{f_{1}^{(\tilde{m})}(\tilde{q})}{f_{1}^{(\tilde{m})}(\tilde{q})}\right)^{8}
$$

where as before $\tilde{m}=\mu p^{+}$. Substituting (4.13) in (4.11) and making the change of variables we find $\int_{0}^{\infty} d l$ times

\footnotetext{
${ }^{9}$ Here we will just quote the results and will not give the details of the computations which can be easily done following [9] and [13].
} 


$$
\tilde{Z}_{\mathcal{A}}=\frac{N^{2}}{8} \int d p^{\prime+} d p^{\prime-} e^{-\pi p^{\prime+} p^{\prime-}} \frac{e^{-2 \pi \mu p^{+}}}{\left(1-e^{-2 \pi \mu p^{\prime+}}\right)^{2}}\left(\frac{f_{1}^{(\tilde{m})}(q)}{f_{1}^{(\tilde{m})}(q)}\right)^{8} .
$$

\section{Mobius Strip}

To compute the Mobius strip amplitude we should make the trace using the Hamiltonian (4.2) over the Hilbert space of open string states projected by the $Z_{2}$ projection (4.3). A straightforward computation gives

$$
Z_{\mathcal{M}}=-\frac{N}{2} \int \frac{d \tau_{2}}{\tau_{2}} \int d p^{+} d p^{-} e^{-2 \pi \tau_{2} p^{+} p^{-}} \mathcal{M}\left(\tau_{2} \mid m\right)
$$

with $t=\tau_{2}$ we have

$$
\mathcal{M}\left(\tau_{2} \mid m\right)=\frac{q^{\frac{m}{2}}}{\left(1-q^{\frac{m}{2}}\right)^{2}}\left(\frac{f_{1}^{\left(\frac{m}{2}\right)}(q) f_{3}^{\left(\frac{m}{2}\right)}(q)}{f_{1}^{\left(\frac{m}{2}\right)}(q) f_{3}^{\left(\frac{m}{2}\right)}(q)}\right)^{8}
$$

We can now perform a modular transformation to go to the transverse channel. In this case we should first take $t^{\prime}=1 / t$ and then $l=t^{\prime} / 2$, this will also change the momentum to $p^{+}=p^{+} / 2 l$ and $p^{-}=2 p^{-}$. The transverse channel amplitude will leads

$$
\tilde{\mathcal{M}}(l \mid \tilde{m})=\frac{e^{-\pi \tilde{m}}}{\left(1-e^{-\pi \tilde{m}}\right)^{2}}\left(\frac{f_{1}^{\left(\frac{\tilde{m}}{2}\right)}(\tilde{q}) f_{3}^{\left(\frac{\tilde{m}}{2}\right)}(\tilde{q})}{f_{1}^{\left(\frac{\tilde{m}}{2}\right)}(\tilde{q}) f_{3}^{\left(\frac{\tilde{m}}{2}\right)}(\tilde{q})}\right)^{8},
$$

where as before $\tilde{m}=\mu p^{+}$. Substituting 4.17 into (4.15) and making the appropriate change of variables we find $\int_{0}^{\infty} d l$ times

$$
\tilde{Z}_{\mathcal{M}}=-\frac{N}{2} \int d p^{\prime+} d p^{\prime-} e^{-\pi p^{++} p^{\prime-}} \frac{e^{-\pi \mu p^{+}}}{\left(1-e^{-\pi \mu p^{+}}\right)^{2}}\left(\frac{f_{1}^{\left(\frac{\tilde{m}}{2}\right)}(\tilde{q}) f_{3}^{\left(\frac{\tilde{m}}{2}\right)}(\tilde{q})}{f_{1}^{\left(\frac{\tilde{m}}{2}\right)}(\tilde{q}) f_{3}^{\left(\frac{\tilde{m}}{2}\right)}(\tilde{q})}\right)^{8}
$$

Note that as for the closed string case each of the three direct channel amplitudes (4.8), (4.12) and (4.16) is zero after the integration over the momentum $p^{+}$and $p^{-}$, due to the fermionic zero modes reflecting the fact that the model is supersymmetric with sixteen supersymmetries 10 i.e. $(8-8)$. However, in the transverse channel The integration over the $p^{+}$ and $p^{-}$momentum should be performed now with some care due to the appearance of a new

\footnotetext{
${ }^{10}$ This is the same as in the flat background where the three amplitudes give zero separately due to the cancellation between the NS-NS and R-R sectors.
} 
singularity $\square$ coming from the factor $\left(1-e^{-\pi \mu p^{+}}\right)^{2}$ which obviously can not be reproduced by a closed-string amplitude calculation being independent of $l$.

We can now study the ultraviolet behavior of the three amplitudes, in the limit $l \rightarrow \infty$ the nontrivial contribution comes from the part which is independent of $l$ (ground state) and we find

$$
\begin{aligned}
\tilde{Z}_{\mathcal{K}}+\tilde{Z}_{\mathcal{A}}+\tilde{Z}_{\mathcal{M}} & =\frac{1}{2} \int d p^{+} d p^{-} \frac{e^{-\pi p^{+} p^{-}}}{\left(1-e^{-\pi \mu p^{+}}\right)^{2}}\left[\left(1+e^{-\pi \mu p^{+}}\right)^{2}+\frac{N^{2} e^{-2 \pi \mu p^{+}}}{4\left(1+e^{-\pi \mu p^{+}}\right)^{2}}-N e^{-\pi \mu p^{+}}\right] \\
& =\frac{1}{2} \int d p^{+} d p^{-} \frac{e^{-\pi p^{+} p^{-}}}{\left(1-e^{-\pi \mu p^{+}}\right)^{2}}\left[\left(1+e^{-\pi \mu p^{+}}\right)-\frac{N e^{-\pi \mu p^{+}}}{2\left(1+e^{-\pi \mu p^{+}}\right)}\right]^{2}
\end{aligned}
$$

It turns out that for $N=8$ the integrand is a regular function of $p^{+}$,

$$
\frac{1}{2} \int d p^{+} d p^{-} e^{-\pi p^{+} p^{-}} \frac{\left(1-e^{-\pi \mu p^{+}}\right)^{2}}{\left(1+e^{-\pi \mu p^{+}}\right)^{2}}
$$

and gives zero by integrating over $p^{+}$and $p^{-}$. Otherwise the integrand is singular and the integral will diverge. Therefore, for the Chan-Paton gauge group $S O(8)$ there is tadpole cancellation. Note that because we are working in the G-S formulation of the theory we are not able to see the vanishing of the NS-NS and R-R tadpoles separately but every thing mix-up in this formalism. In [15] the authors proposed a way to read off the contribution of the R-R states by inserting a projection operator $\frac{1-(-1)^{F_{L}}}{2}$ in the tree and one loop channel amplitudes. We have not attempted to do this in the present work.

\section{Conclusion}

In this paper we have computed the partition function of type IIB string theory in plane wave R-R 5-form background using both path integral and operator formalisms. We have shown that the two give the same result and is equal to the partition function of type IIB theory in flat background. The same observation apply to type IIB in plane wave NS-NS and R-R 3-form backgrounds of [1]. The partition function of the NW model was computed in [12 and found to be equal to that of flat space. It would be very interesting to determine the massless vertex operators and compute correlation functions in the plane wave $\mathrm{R}-\mathrm{R}$ background. The one-loop amplitudes will be important for the comparison with the non-planar diagrams in the gauge theory.

We have studied the tadpole cancellation in a model of unoriented closed and open strings in plane wave background dual to $\mathcal{N}=2 S p(N)$ gauge theory with hypermultiplet in the antisymmetric representation and four fundamental hypermultiplets. We have seen that there is tadpole cancellation for $\mathrm{SO}(8)$ gauge group. It will be very interesting to have a NSR formulation of string theory in plane wave R-R background.

\footnotetext{
${ }^{11} \mathrm{~A}$ similar factor was analysed in 15 contributing $\frac{1}{2 \pi \tilde{m}}$ that was interpreted as a volume factor.
} 


\section{Acknowledgments:}

I am very greatfull to Edi Gava, Matthias Blau, Nouraddine Chair, Kumar S. Narain, Jorge G. Russo, George Thompson, Elias Kiritsis and Jose F. Morales for very illuminating discussions. I would like to thank the High Energy Group of the Abdus Salam International Center for Theoretical Physics for Hospitality and Grants. This work is partially supported by ATN contract: HPRN-CT-2000-00122.

\section{References}

[1] M. Blau, J. Figueroa-O'Farrill, C. Hull and G. Papadopoulos, "A new maximally supersymmetric background of IIB superstring theory," JHEP 0201 (2002) 047, hep-th/0110242.

[2] M. Blau, J. Figueroa-O'Farrill, C. Hull and G. Papadopoulos, "Penrose limits and maximal supersymmetry," Class. Quant. Grav. 19 (2002) L87, hep-th/0201081; M. Blau, J. Figueroa-O'Farrill and G. Papadopoulos, "Penrose limits, supergravity and brane dynamics," hep-th/0202111.

[3] R. R. Metsaev, "Type IIB Green-Schwarz superstring in plane wave Ramond-Ramond background," Nucl. Phys. B 625 (2002) 70, hep-th/0112044; R. R. Metsaev and A. A. Tseytlin, "Exactly solvable model of superstring in plane wave Ramond-Ramond background," hep-th/0202109.

[4] J.G. Russo and A.A. Tseytlin "On solvable models of type 2B superstring in NS-NS and R-R wave backgrounds", JHEP 0204 (2002) 021, hep-th/0202179.

[5] D. Berenstein, J. M. Maldacena and H. Nastase, "Strings in flat space and pp waves from N = 4 super Yang Mills," JHEP 0204 (2002) 013, hep-th/0202021.

[6] D. Berenstein, E. Gava, J. M. Maldacena, K. S. Narain and H. Nastase, "Open Strings On Plane Waves and Their Yang-Mills Duals," hep-th/0203249.

[7] A. Fayyazuddin and M. Spalinski, "Large N Superconformal Gauge Theories and Supergravity Orientifolds", Nucl. Phys. B535 (1998) 219, hep-th/9805096; O. Aharony, A. Fayyazuddin and J. Maldacena, "The Large $\mathrm{N}$ limit of $\mathcal{N}=1,2$ from Three-Branes in F-Theory", JHEP 9807(1998) 013, hep-th/9807159.

[8] A. A. Tseytlin, unpublished work.

[9] T. Takayanagi, "Modular invariance of strings on pp-waves with RR-flux," hep-th/0206010.

[10] R. Kallosh and A. Morozov, "Green-Scwarz Action and Loop calculation for Superstrings," Int.J.Mod.Phys. A 3(1988)1943-1958.

[11] B. Craps, D. Kutasov, G. Rajesh, "String Propagation in the Presence of Cosmological Singularities", JHEP 0206 (2002) 053, hep-th/0205101. 
[12] E. Kiritsis, C. Kounnas, "String Propagation in Gravitational Wave Background," Phys.Lett. B 320:264-272,1994, hep-th/9310202. E. Kiritsis, C. Kounnas, D. Lust, "Superstring Gravitational Wave Backgrounds with Space-Time Supersymmetry," Phys.Lett. B 331:321-329,1994, hep-th/9404114. E. Kiritsis, B. Pioline, "Strings in homogeneous gravitational waves and null holography," JHEP 0208 (2002) 048, hep-th/0204004.

[13] O. Bergman, M. R. Gaberdiel and M. B. Green, "D-brane interactions in type IIB planewave background," hep-th/0205183.

[14] C. Angelantonj and A, Sagnotti, "Open Strings," hep-th/0204089.

[15] A. Sinha, N. V Suryanarayana, "Tadpole Analysis of Orientifolded Plane-Waves," hepth/0209247. 